\title{
Pseudotumor renal secundario a pancreatitis aguda. A propósito de un caso
}

\author{
Ll. Fumadó Ciutat, M.Á. López-Costea, F.J. Elías Cañavera, O. Buisán Rueda, \\ L. Riera Canals, E. Franco Miranda
}

Servicio de Urología. Hospital Universitario de Bellvitge. L'Hospitalet de Llobregat (Barcelona)

Actas Urol Esp 2005; 29 (8): 794-796

\section{RESUMEN}

PSEUDOTUMOR RENAL SECUNDARIO A PANCREATITIS AGUDA. A PROPÓSITO DE UN CASO.

Se presenta el caso de un pseudotumor renal correspondiente a un granuloma esteatonecrótico, secundario a una pancreatitis aguda enólica en los meses previos. Las características de los pseudotumores renales, su diagnóstico y la actitud terapéutica son discutidos con el caso de un varón de 60 años que presenta una masa renal hallada incidentalmente en una tomografia computerizada abdominal.

Palabras clave: Pseudotumor renal. Granuloma esteatonecrótico. Pancreatitis aguda. Cirugía parcelar renal.

\section{ABSTRACT}

RENAL PSEUDOTUMOR SECONDARY TO ACUTE PANCREATITIS. A CASE REPORT

We present the case of a renal pseudotumor corresponding to a esteatonecrotic granuloma, secondary to an enolic acute pancreatitis a few months ago. The main caractheristics, diagnosis and terapeuthics of the renal pseudotumors are discussed with the case report of a 60 year old man who presents a renal mass founded incidentally at a control computed tomography.

Keywords: Renal pseudotumor. Esteatonecrotic granuloma. Acute pancreatitis. Nephron sparing surgery.

$\mathrm{L}$ a proliferación en la realización de pruebas de imagen para el estudio de patologías diversas ha provocado el aumento en la detección de masas renales incidentales, hasta el punto de significar la forma de presentación más frecuente del cáncer renal, superando el 50\% en algunas series $^{1,2,3}$.

Aun así, de todas las masas renales extirpadas con criterios de malignidad, un $10-15 \%$ resultarán ser tumoraciones benignas: entre el $3 \mathrm{y}$ el $7 \%$ corresponden a oncocitomas ${ }^{4,5}$, entre $0,7 \mathrm{y}$ $2 \%$ a angiomiolipomas ${ }^{6}$, y entre el 5 y el $8 \%$ corresponden a masas renales indetermidadas o pseudotumores $^{7,8}$. Estas últimas incluyen los quistes renales complejos (quistes hemorrágicos, infectados, hiperdensos, nefroma quístico multilocular, carcinomas quísticos), la nefronia lobar, los procesos inflamatorios, el infarto renal, el teji- do renal anómalo (columna de Bertin hipertrofiada) y las masas renales de pequeño tamaño. Los procesos inflamatorios del tejido perirrenal que plantean el diagnóstico diferencial con un cáncer renal son, en la mayoría de los casos, secundarios a pielonefritis xantogranulomatosas, pielonefritis focales o segmentarias.

El caso que nos ocupará a continuación se presenta como hallazgo concomitante y a distancia de una pancreatitis aguda enólica.

\section{CASO CLÍNICO}

Paciente varón de 60 años de edad, fumador, enolismo severo, que presenta como antecedentes patológicos hipertensión arterial y episodio de pancreatitis aguda enólica en noviembre del 2003, en cuyo ingreso se realizó TC abdominal que objetivó inflamación pancreática difusa con 
exudados, así como la presencia de dos tumoraciones en valva anterior de riñón izquierdo (Fig. 1), una de ellas de características tomodensitométricas que sugerían un quiste hemorrágico o con alto contenido proteico, sin poder descartar una tumoración sólida.

Tras la recuperación del cuadro inflamatorio agudo se comentó el caso con nosotros, y continuamos el estudio de dicha tumoración mediante la realización de nuevo TC abdominal de control en febrero 2004 (Fig. 2), observando la persistencia de ambas lesiones renales. Dadas las características radiológicas de la tumoración indicamos la intervención quirúrgica. El día 29 de marzo del 2004 realizamos tumorectomía renal izquierda vía lumbotomía.

El resultado de anatomía patológica de la pieza resultó ser granuloma esteatonecrótico con abundante pigmento férrico, característico de las necrosis grasas secundarias a pancreatitis.

\section{DISCUSIÓN}

La pancreatitis aguda es un proceso inflamatorio de gran intensidad que provoca la destrucción de los tejidos adyacentes por activación de los enzimas pancreáticos, siendo frecuente la desestructuración de la grasa peripancreática y la presencia de abundantes exudados, con la formación de pseudoquistes pancreáticos que pueden afectar a

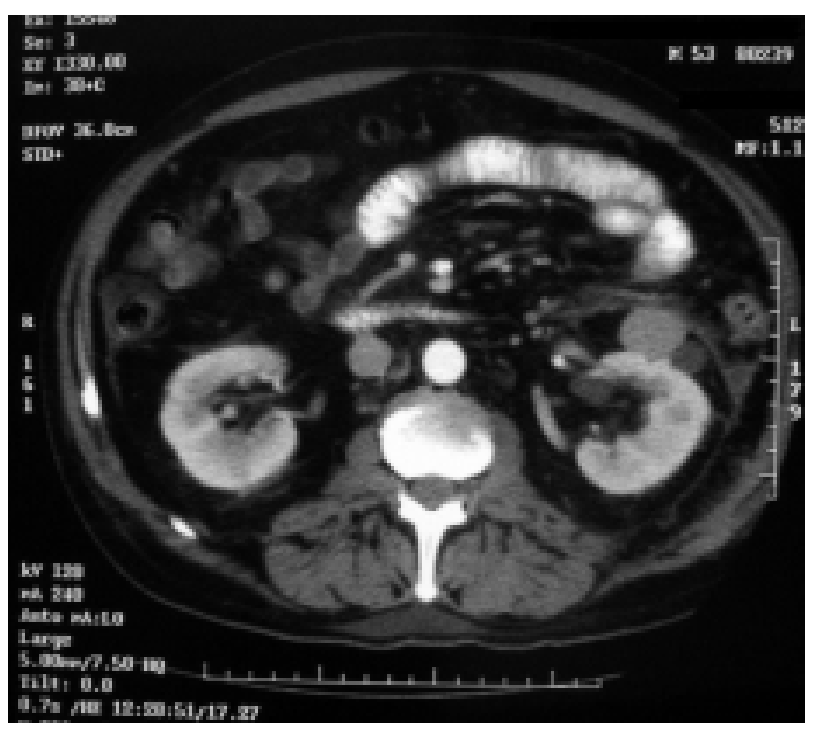

FIGURA 1. Se observan las dos lesiones en valva anterior del riñón izquierdo, halladas incidentalmente en el estudio de la pancreatitis aguda

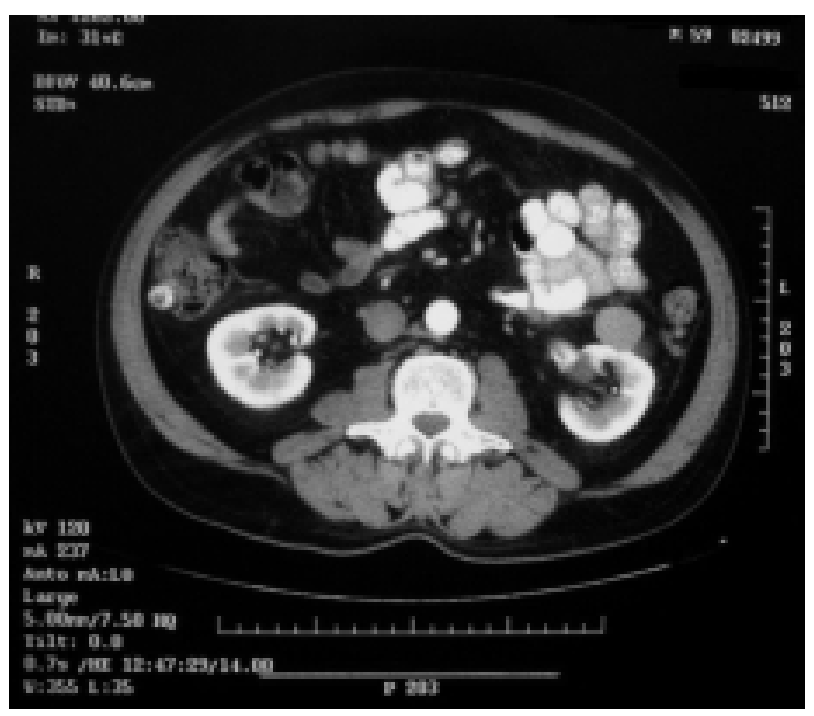

FIGURA 2. A los tres meses se repitió el TC abdominal, evidenciando la persistencia de las lesiones, sin poder descartar la malignidad de la lesion sólida.

los órganos vecinos. El bazo y el estómago son los órganos más frecuentemente implicados.

La afectación renal suele ser la insuficicencia renal aguda, causada inicialmente por hipovolemia efectiva debida al tercer espacio que condiciona la inflamación aguda, y secundariamente parenquimatosa por necrosis tubular debida a radicales libres oxidativos.

Paralelamente se puede producir una afectación renal directa por progresión del proceso inflamatorio, si bien, es mucho menos frecuente. Hay casos descritos en la literatura de presentación aguda como síndrome de Wünderlich ${ }^{9}$, así como la combinación de pancreatitis aguda y masa renal concomitante ${ }^{10,11}$ debidas a carcinoma de celulas renales. Sin embargo, no hay evidencia publicada de ningún caso de granuloma esteatonecrótico renal secundario a pancreatitis aguda.

La pancreatitis aguda puede ser una forma de presentación del cáncer renal ${ }^{10,11}$, y a la inversa una inflamación pancreática puede comprometer físicamente al rinón.

La actitud terapéutica no diferirá respecto a los casos aislados ${ }^{12}$ : en caso de ser quísticas, según la clasificación de Bosniak ${ }^{13,14}$ no será necesario tratamiento ni seguimiento para las lesiones tipo I. Las lesiones tipo II deberán ser controladas periódicamente, y las tipo III y IV son indicación de exéresis quirúrgica. En caso de 
tratarse de una lesión sólida, se debe considerar maligna hasta que no se demuestre lo contrario.

En el caso que nos ocupa la lesión cumplía criterios de malignidad y dado que el único tratamiento curativo para el cáncer renal es la exéresis quirurgica ${ }^{15}$, se procedió a la intervención quirúrgica.

En este contexto, la cirugía ahorradora de nefronas o cirugía parcelar renal cobra especial protagonismo en la exéresis de masas renales, principalmente por la posibilidad de "sobretratar" una masa renal con cirugía radical cuando en un 10-15\% de los casos resultará ser una masa renal benigna.

La selección de los pacientes deberá ser cuidadosa. Serán candidatos a cirugía conservadora los tumores renales que cumplan todos los criterios siguientes ${ }^{16}$ :

- Tamaño menor de $4 \mathrm{~cm}$.

- Localización periférica

- No invasión del sistema calicial

- No adenopatías

- No invasión extrarrenal

- No hematuria macroscópica

Los casos en los que la nefrectomía aboque indefectiblemente a la insuficiencia renal a corto o medio plazo también serán candidatos a cirugía conservadora $^{16}$.

Todo ello refuerza la opción de la cirugía parcial en aquellos tumores que cumplan los criterios antes mencionados.

\section{REFERENCIAS}

1. Belldegrun A, De Kernion JB. Renal Tumors; In: Campbell's Urology, Ed. Walsh, P.C., Retik, A.B., Vaughan E.D., Wein, A.J., W.B. Saunders, Philadelphia 1998;2283-2326.

2. Porena M, Velpasiani G, Rosi P, et al. Incidentally detected renal cell carcinoma: role of Ultrasonography. J Clin Ultrasound 1992;20:395-400.
3. Herranz Amo F, Jara Rascon J, Lledo Garcia E, et al. Estudio clínico de los adenocarcinomas renales diagnosticados de forma incidental. Arch Esp Urol 1995;48:991-998.

4. Lieber MM. Renal Oncocytoma. Urol Clin North Am 1993; 20:355-359.

5. Davis CJ, Mostofi FK, Sesterhenn IA, et al. Renal oncocytoma: clinicopathological study of 166 pacients. J Urogenital Pathol 1991;1:41-52.

6. Peligros Gómez MI, Álvarez Fernández E. Anatomía patológica de los tumores del riñón del adulto; En : Herranz Amo, F.: Tumores del riñón, Ed. Ed Doyma, 2001: 27-39.

7. Balfe DM, Mcclennan BL, Stanley RJ et al. Evaluation of renal masses considered indeterminate on computed tomography. Radiology 1982;142:421-428.

8. Curry NS, Reinig J, Schabel SI, et al. An evaluation of the effectiveness of ct vs. other imaging modalities in the diagnosis of atypical renal masses. Invest radiol 1984;19: 447454

9. Albi G, Del Campo L, Tagarro D. Wündelich's Syndrome: Causes, diagnosis and radiological management. Clin Radiol 2002;57:840-845.

10. Gibo J, Ito T, Kawab K, et al. A case of acute pancreatitis induced by metastasis of renal cell carcinoma. Nippon Shokakibyo Gakai Zarshi 2003;100:901-906.

11. Nabi G, Dogra PN, Chowdhairy A. Renal cell carcinoma presenting as acute pancreatitis. Urol Int 2002;68:202203.

12. Wolf JS JR. Evaluation and management of solid and cystic renal masses. J Urol 1998;159:1120-1133.

13. Bosniak MA. The current radiological aproach to renal cyst. Radiology 1986;158:1-10.

14. Bosniak MA. Diagnosis and management of patients with complicated renal cystic lesions of the kidney. Am J Roentgenol 1997;169:819-821.

15. Godley PA, Stinchcombe TE. Renal cell carcinoma. Curr Opin Oncol 1999;11:213-217.

16. López-Costea MA, González-Satué C, Franco Miranda E, et al. Nefrectomía parcial en el carcinoma de celulas renales. Actas Urol Esp 2001;25:482-488.

Dr. Ll. Fumadó Ciutat

Servicio de Urología. Hospital de Bellvitge

Feixa Llarga, s/n

08907 Hospitalet de Llobregat (Barcelona)

37615lfc@comb.es

(Trabajo recibido el 4 enero 2005) 